\title{
GADAMERS VERSTÄNDNIS UND MISSVERSTÄNDNIS VON SCHLEIERMACHER
}

\section{BEN VEDDER}

To cite this article: BEN VEDDER (2003) GADAMERS VERSTÄNDNIS UND

MISSVERSTÄNDNIS VON SCHLEIERMACHER, Bijdragen, 64:4, 400-420, DOI: 10.2143/

BIJ.64.4.503584

To link to this article: https://doi.org/10.2143/BIJ.64.4.503584

册 Published online: 25 Apr 2013.

Submit your article to this journal $\pi$

Llll Article views: 11

Q View related articles $\sqsubset$

Citing articles: 1 View citing articles $\square$ 


\section{GADAMERS VERSTÄNDNIS UND MISSVERSTÄNDNIS VON SCHLEIERMACHER}

\section{BEN VEDDER}

Das Thema dieses Artikels ist Gadamers Interpretation von Schleiermacher. Es scheint als kenne Gadamer in Wahrheit und Methode Schleiermacher vor allem aus Diltheys Darstellung von Schleiermacher. ${ }^{1}$ Ob Gadamer Schleiermacher richtig verstanden hat, ist sehr zweifelhaft; schon seine ersten Verweisungen auf Schleiermacher sind sehr deutlich von Dilthey inspiriert worden: "Schleiermacher, mit dessen hermeneutischer Theorie wir uns später noch beschäftigen werden, ist ganz darauf gerichtet, die ursprüngliche Bestimmung eines Werkes im Verständnis wiederherzustellen. Denn Kunst und Literatur, die uns aus der Vergangenheit überliefert sind, sind ihrer ursprünglichen Welt entrissen." (WuM, 158) Dies klingt sehr nach Dilthey. Wenn wir Gadamers Beschreibung von Schleiermachers Hermeneutik genauer ansehen, dann fasst Gadamer dies in großen Linien wie folgt zusammen. Schleiermacher sucht in seiner Schrift über Hermeneutik die theoretische Begründung des den Theologen und Philologen gemeinsamen Verfahrens zu gewinnen, indem er hinter beiden Anliegen auf ein ursprünglicheres Verhältnis des Verstehens von Gedanken zurückgeht. Die Bemühung des Verstehens findet überall dort statt, wo sich kein unmittelbares Verstehen ergibt, bzw. wo mit der Möglichkeit eines Missverstehens gerechnet werden muss. (WuM, 167) Diese Möglichkeit des Missverständnisses ist mit der Individualität des Anderen verbunden. In einem neuen, universellen Sinn ist Fremdheit mit der Individualität des Du unauflöslich gegeben. (WuM, 168) Zu Recht verweist Gadamer darauf, dass die Unverständlichkeit bei Schleiermacher eine universelle Bedeutung bekommt, er spricht aber nicht von einem Unverständnis, sondern vom Missverständnis. (WuM, 173)

Die Praxis der Hermeneutik besteht darin, Missverständnisse zu vermeiden. Hermeneutik erhebt sich über die pädagogische Okkasionalität der Auslegungspraxis zur Selbständigkeit einer Methode, sofern “das Missverstehen

\footnotetext{
${ }^{1}$ Hans Georg Gadamer, Wahrheit und Methode. Grundzüge einer philosophischen Hermeneutik, Tübingen ${ }^{4} 1975$, S. 60. Im Folgenden im Text abgekürzt als WuM.
} 
sich von selbst ergibt und das Verstehen auf jedem Punkt (...) gewollt und gesucht werden" muss. (WuM, 173) ${ }^{2}$ Dazu entwickelt Schleiermacher einen Kanon grammatischer und psychologischer Auslegungsregeln, die sich vor aller dogmatisch-inhaltlichen Bindung im Bewusstsein des Auslegers niederzuschlagen haben. Es ist Schleiermacher, der die Hermeneutik zu einer universellen Hermeneutik ausarbeitet (WuM, 172). Gadamer behauptet, dass das äußere Wort mit dem inneren Wort verwoben ist, und dass alle Probleme der Auslegung in Wahrheit Probleme des Verstehens sind. (WuM, 173) Im Folgenden werde ich zeigen, dass das Umgekehrte ebenso gilt: die Probleme des Verstehens sind am Ende auch Probleme der Auslegung und der Sprache - ein Problem, das Gadamer zuwenig bedacht hat.

In der Hermeneutik geht es darum, das in Rede und Text Gemeinte verständlich zu machen. Die Kunst der Hermeneutik ist nie das Organon der Sachforschung gewesen. Das unterscheidet sie von jeher von dem, was Schleiermacher Dialektik nennt. Doch weist Gadamer daraufhin, dass indirekt überall da, wo man sich um das Verständnis - z. B. der Heiligen Schrift oder der Klassiker - bemüht, ein Bezug auf die Wahrheit wirksam ist, die im Text verborgen liegt und ans Licht gebracht werden soll. Obwohl Gadamer weiß, dass bei Schleiermacher eine enge Verbindung von Hermeneutik und Dialektik besteht (WuM, 174), weist in seiner SchleiermacherInterpretation nichts auf diese Beziehung hin. Und die Bezogenheit auf die Wahrheit in der Hermeneutik hätte Gadamer in Schleiermachers Dialektik finden können.

Im Aufsatz "Hermeneutik und Historismus", der in der zweiten Auflage von "Wahrheit und Methode" im Anhang veröffentlicht wurde, hält Gadamer an seiner Meinung fest, ungeachtet der Ergebnisse neuerer SchleiermacherPublikationen von Kimmerle und Redeker. "Schleiermachers eigenster Beitrag war dabei die psychologische Interpretation, wonach jeder Gedanke eines Textes als ein Lebensaugenblick auf den persönlichen Lebenszusammenhang seines Verfassers zurückbezogen werden muss, wenn er ganz verstanden werden will." (WuM, 493 f.) Die Forschungsergebnisse der Schleiermacher-Forschung nach dem Erscheinen von "Wahrheit und Methode" bewegen Gadamer nicht zu einer Revision seines früheren Urteils. Auch wenn er 1968 eigens einen Aufsatz über Schleiermacher schreibt, bleibt er, sieht man von einigen Nuancierungen $a b$, bei seiner Betonung der psychologischen Interpretation. Die Rede ist vom Aufsatz "Das Problem der Sprache

${ }^{2}$ Gadamer zitiert Schleiermachers Hermeneutik, $\S 15$ und 16, Werke I, 29 f. 
in Schleiermachers Hermeneutik" 3 . Laut Gadamers eigenem Urteil ist dieser Aufsatz dem Reichtum des individualisierenden Verstehens bei Schleiermacher besser gerecht geworden als "Wahrheit und Methode". (GW 2, 15) Auch später bleibt Gadamer bei seiner Meinung, dass "nicht in der grammatischen, sondern in der psychologischen Interpretation [...] die Individualisierung und damit das hermeneutische Problem" steckt. (GW 2, 15, Fußnote) Er bemerkt diese Nachlässigkeit bei Schleiermacher, wenn er sich mit der Position von Manfred Frank auseinandersetzt. (GW 2, 14) Frank kritisiert Gadamers psychologische Schleiermacher-Auslegung ${ }^{4}$ mit strukturalistischen Überlegungen, und zwar sowohl mit Bezug auf die psychologische Interpretation als auch mit Bezug auf den Begriff der Divination. Laut Frank kommt es darauf an, Schleiermacher in Konfrontation mit seinem eigenen Anliegen zu kritisieren. Gadamer erkennt zwar, dass er die Dialektik Schleiermachers stärker hätte beachten sollen, ohne jedoch daraus Konsequenzen für seine Schleiermacher-Interpretation zu ziehen. Wie ich nachweisen werde, hat Gadamer nicht erkannt, dass die Dialektik den Zugang zu Schleiermachers Hermeneutik bildet. $\mathrm{Ob}$ dies eine Folge des phänomenologischen Ansatzes ist, den er vom frühen Heidegger übernimmt, lasse ich hier ununtersucht. Obwohl Gadamer seine Schleiermacher-Interpretation später korrigiert hat, bleibt er seiner ursprünglich psychologischen Interpretation treu. Meines Erachtens ist dies eine Folge davon, dass Gadamer Schleiermachers Dialektik nicht angemessen berücksichtigt. Aus diesem übersieht er eine Verwandtschaft zwischen seiner Hermeneutik und der von Schleiermacher.

Wir werden sehen, dass bei Schleiermacher die Hermeneutik als ein Ganzes von Regeln wichtig für die Erkenntnissicherheit ist. Im Gegensatz zu Gadamers Interpretation wird diese Sicherheit jedoch nie vollends in der Hermeneutik erreicht. Daher hat die Hermeneutik bei Schleiermacher immer etwas Vorläufiges, sie kann sich nie auf einen absoluten Standpunkt stellen. Im Folgenden möchte ich mich zuerst mit den hermeneutischen Einsichten Schleiermachers auseinandersetzen. Danach werde ich auf die Beziehung zwischen Hermeneutik und Dialektik eingehen. Anschließend interessieren mich erneut die Aufgabe, Grenzen und Endlichkeit der Hermeneutik in Schleiermachers Ausführungen, die ich mit Gadamers Verständnis, aber auch Missverständnis konfrontieren werde.

\footnotetext{
${ }^{3}$ Ders., Gesammelte Werke 4, Tübingen 1987, 361-373. Im Folgenden abgekürzt als GW 4.

${ }^{4}$ Manfred Frank, Das individuelle Allgemeine. Textstrukturierung und Interpretation nach Schleiermacher, Frankfurt 1977.
} 


\section{Schleiermachers Begriff der Hermeneutik}

In "Wahrheit und Methode" fasst Gadamer Schleiermachers Hermeneutik wie folgt zusammen: "Wiederherstellung der 'Welt', der es zugehört, Wiederherstellung des ursprünglichen Zustandes, den der schaffende Künstler 'gemeint' hatte.[...] Das ist in der Tat der Schleiermachersche Gedanke, den seine gesamte Hermeneutik stillschweigend voraussetzt [...] Genau wie es sonst Texten gegenüber verfährt, indem es die ursprüngliche Produktion des Verfassers zu reproduzieren strebt." (WuM, 159) Von der "gesamten Hermeneutik" zu reden, ist insofern nicht unproblematisch, wenn man bedenkt, dass Schleiermacher zu seinen Lebzeiten nur wenig zum Thema Hermeneutik publiziert hat. Auf diese wenigen Quellen stützt sich Gadamers Lektüre Schleiermachers ${ }^{5}$, dessen Theorie der Hermeneutik auf einer Grundeinsicht beruht: Jedes Verstehen fremden Sprechens gehört zum Bereich der Hermeneutik. Dies bedeutet, dass die Hermeneutik weder die exklusive Domäne der Bibelauslegung in der Theologie ist, noch auf die zum Studium der antiken Literatur notwendigen Auslegungsmethoden beschränkt ist. Der ganze Bereich des Sprechens und der Sprache gehört zur Hermeneutik; Hermeneutik ist da, wo gesprochen wird und wo Schriftsteller und Leser sind. Daher können die Prinzipien der Hermeneutik im ganzen Sprachbereich angewendet werden, und nicht nur im Bereich der antiken Literatur und der Bibel. Hermeneutik ist so universell wie die Sprache.

Die wichtigste Voraussetzung für die Hermeneutik ist das Fremde, das verstanden werden soll. Außerdem muss es auch etwas Vertrautes oder Gemeinschaftliches am gesprochenen Wort oder Text, der ausgelegt werden soll, geben, da sonst ein Verstehen des Fremden unmöglich wäre. Ist etwas völlig fremd, ist keine Auslegung möglich, und wenn etwas bereits allseits bekannt ist, wäre eine Auslegung überflüssig. Im diesem Fall brauchte man einen Text nur zu hören bzw. zu lesen, um ihn zu verstehen. In Schleiermachers Verständnis ist die Hermeneutik zwischen Fremdheit und Vertrautheit, zwischen Unwissenheit und Bekanntheit situiert. Hermeneutik erstreckt sich auf all diejenigen Sprachgebiete, in denen das Ausdrücken von Gedanken für den Zuhörer bzw. Leser etwas Fremdes oder Unbegreifliches ist, das nur dann

\footnotetext{
${ }^{5}$ Diese Ausführungen stützen sich vor allem auf Schleiermachers "Über den Begriff der Hermeneutik, mit Bezug auf F.A. Wolfs Andeutungen und Asts Lehrbuch". Dies ist der einzige Text, den Schleiermacher zu seinen Lebzeiten über das Thema Hermeneutik publiziert hat. Ich zitiere nach: Fr. D. E. Schleiermacher, Hermeneutik, nach den Handschriften neu herausgegeben und eingeleitet von Heinz Kimmerle, Heidelberg 1959 (abgekürzt als HK).
} 
verstanden oder begriffen werden kann, wenn der Hörende etwas von dem, was der Sprechende sagt, versteht. Gleiches trifft auf den Lesenden zu.

Hermeneutik hat für Schleiermacher mit dem alläglichen Sprechen und Schreiben zu tun, nicht nur mit klassischen oder theologischen Schriften. Dies gibt Schleiermacher die Möglichkeit, das Modell des Gesprächs als hervorragendes Modell auf die Hermeneutik anzuwenden, ebenso auf die Hermeneutik der geschriebenen Sprache. Hermeneutik hat ihren Platz vor allem im Gespräch; sie vollzieht sich als Gespräch. (HK, 129 f.) Das ist ein Gedanke, den Gadamer aufnimmt und der bei ihm weiterwirkt. Über die Wirkung schreibt Gadamer selbst 1968: "Aber im Hintergrunde der Schleiermacherschen Konzeption einer allgemeinen Hermeneutik stand [...] ein philosophisches Motiv. Einer der tiefsten Antriebe des romantischen Zeitalters war der Glaube an das Gespräch als eine eigene, undogmatische und durch keine Dogmatik ersetzbare Wahrheitsquelle." (GW 2, 97)

Laut Gadamer hat Schleiermacher der hermeneutischen Reflexion ein grundsätzliches Motiv gegeben, um das Problem der Hermeneutik in einen Horizont zu stellen, wie ihn die bisherige Hermeneutik nicht kannte. (WuM, 168) Es ist diese Konstellation, die Schleiermachers Stellung in der Hermeneutik verständlich mache: "Wir gehen von dem Satz aus: Verstehen heißt zunächst, sich miteinander verstehen. Verständnis ist zunächst Einverständnis [...] Verständigung ist also immer: Verständigung über etwas. Sich verstehen ist Sichverstehen in etwas." (WuM, 168) Wenn Gadamer auf den dialogischen Charakter der Hermeneutik verweist, den Schleiermacher erkannt hat, so ist diese Bemerkung nicht ganz korrekt. Denn Schleiermachers Hermeneutik ist im Grunde eine Analyse des Gesprächs.

Schleiermacher selbst ertappt sich regelmäßig im Gespräch bei hermeneutischen Operationen, wenn er die Gedanken seines Gesprächspartners miteinander zu verbinden versucht. Er definiert die hermeneutische Operation als ein Miteinander-Verbinden von Gedanken - ein Vorgang, der nicht notwendig voraussetzt, dass die Sprache, in der das Gespräch geführt wird, eine fremde Sprache ist; auch beim Gebrauch derselben Sprache kann es Momente des Missverstehens geben. Hermeneutik ist ein inhärenter Bestandteil der Sprache und Kultur als solcher. Schleiermachers Vorliebe für die gesprochene Sprache entspringt dem Gedanken, dass das Gespräch eine Gedankenkette manifestiert, die Ausdruck des Lebens eines konkreten Individuums. Diese Relation zwischen den jeweiligen Gedanken und dem individuellen Lebensganzen wird in der geschriebenen Sprache nicht erkannt oder leicht übersehen. Jede Aussage bleibt ja von anderen Lebensumständen umgeben. 
Um Missverständnisse auflösen zu können, die die Folge menschlichen Sprechens sind, sucht Schleiermacher nach einer für die Hermeneutik eigentümlichen Sicherheit. Schleiermacher sowohl bei der historisch-grammatischen als auch bei der psychologisch-technischen Hermeneutik vom Interpreten eine gute Fertigkeit und Kenntnis. Eine Besonderheit bildet die psychologische Hermeneutik insofern, als hier nicht von jedem Interpreten die gleichen Fertigkeiten verlangt werden können. Aus diesem Grund teilt Schleiermacher die Hermeneutik in zwei Gebiete: einen grammatischen Bereich, worin Sprache und Geschichte studiert wird, und in einen psychologisch-technischen Bereich, in dem die Sprache als Ausdruck des persönlichen Lebens zu studieren ist. Ersteres verleiht der Hermeneutik eine objektive Ausrichtung, letzteres eine subjektive.

Neben diesen unterschiedlichen Methoden muss besonders das hervorgehoben werden, was Schleiermacher eine 'divinatorische' Sicherheit nennt. Diese Art Sicherheit ist eine suchende oder vermutende Sicherheit. Man kann z.B. beweisen, dass ein Wort innerhalb bestimmter Kombinationen von Wörtern diese spezifische Bedeutung hat, aber es gibt auch viele Texte, über die man nur wenig mit einiger Sicherheit sagen kann. Diese Unsicherheit findet sich sowohl in der grammatischen als auch in der psychologischen Hermeneutik. In diesem Fall muss der Übersetzer die Bedeutung des Textes an dieser bestimmten Stelle erahnen. Welche Bedeutung eine Textstelle hat, ergibt sich aus der geschichtlichen Situation des Autors, der dem Text einen individuellen Charakter durch die Art verleiht, wie er die Wörter kombiniert und zusammenfügt. Geschichtlich situiert sind nicht nur die subjektive Meinung und die Gedanken des Autors bzw. Sprechers, sondern auch die objektive Verbindung von Wörtern und Sätzen. Auf diesem Gebiet ist zwischen Behauptung und Beweis zu differenzieren, insofern steht die divinatorische Sicherheit im Gegensatz zur demonstrativen Sicherheit, die das Ergebnis einer vergleichenden Forschung ist.

Bei Gadamer wird diese divinatorische Sicherheit mit der psychologischen Hermeneutik in einen Zusammenhang gebracht, wie er sich bei Schleiermacher nicht findet. Die Einteilung in grammatische und psychologische Hermeneutik ist nicht mit der Einteilung in demonstrative und divinatorische Sicherheit zu verwechseln. In beiden Bereichen der Hermeneutik gibt es demonstrative und divinatorische Sicherheit. Für die erste ist der Vergleich charakteristisch, für die zweite die Ahnung und Vermutung. Zwischen der vergleichenden und der divinatorischen Methode muss es einen Zusammenhang geben. Bei der vergleichenden Methode bringt der Interpret das Bekannte und Vertraute mit dem Unbekannten und Fremden in Beziehung. Auf diese Weise wird er das 
Unbekannte immer enger einkreisen. Der Vergleich lässt jedoch die Erklärung nicht völlig überflüssig werden. Im Gegenteil: die divinatorische Methode bleibt notwendig, da es denkbar ist, dass der Autor bzw. Sprecher von ihren grammatischen Regeln und gewohntem Standpunkt abweichen kann.

Nun versteht Gadamer Schleiermacher so, dass zu dem, was verstanden werden soll, nicht nur der Wortlaut und sein objektiver Sinn gehört, sondern auch die Individualität des Sprechenden bzw. des Verfassers. Schleiermacher ist der Ansicht, dass diese Individualität sich nur im Rückgang auf die Entstehung der für den Verfasser eigentümlichen Gedanken wirklich verstehen lässt. (WuM, 174) Die von Gadamer kritisierte psychologische Hermeneutik (Ebd.) scheint in Schleiermachers Gedanken eines Verstehens der Individualität angelegt zu sein: "Schleiermachers Eigenstes ist aber die psychologische Interpretation. Sie ist letzten Endes ein divinatorisches Verhalten, ein Sichversetzen in die ganze Verfassung des Schriftstellers, eine Auffassung des 'inneren Herganges' der Abfassung eines Werkes, ein Nachbilden des schöpferischen Aktes." (WuM, 175) Gadamer betont: "Auf alle Fälle ist diese psychologische Interpretation für die Theorienbildung des 19. Jahrhunderts für Savigny, Boeckh, Steinthal und vor allem Dilthey - die eigentlich bestimmende geworden." (WuM, 175) Wie diese beiden Zitate belegen, identifiziert Gadamer das psychologische mit dem divinatorischen Verstehen, ohne zu erkennen, dass das psychologische Verstehen ebenso komparativ wie das grammatische Verstehen ist.

Gadamer benutzt Schleiermacher quasi wie einen Sparringpartner, an dem er die Vorzüge seines hermeneutischen Ansatzes demonstriert: "Was Schleiermacher als subjektive Interpretation entwickelt hat, darf wohl ganz beiseite gesetzt werden. Wenn wir einen Text zu verstehen suchen, versetzen wir uns nicht in die seelische Verfassung des Autors, sondern wenn man schon von Sichversetzen sprechen will, so versetzen wir uns in die Perspektive, unter der der andere seine Meinung gewonnen hat. Das heißt aber nichts anderes, als das wir das sachliche Recht dessen, was der andere sagt, gelten zu lassen suchen." (WuM, 276)

Die Notwendigkeit der divinatorischen Methode erklärt Schleiermacher aber nicht aus der Subjektivität des Autors oder des Sprechers, sondern aus dem Faktum, dass Denken nie ohne Wörter stattfindet. Die Tatsache, dass Denken (universell) in Wörtern (geschichtlich) stattfindet, macht den Gebrauch beider Methoden notwendig. Bei Schleiermacher ist die Beziehung zwischen Wörtern und Denken zirkulär; es ist eine Beziehung wechselseitiger Implikation. Um dies deutlich zu machen, verweist Schleiermacher auf die Situation des Kindes, das sprechen lernt. (HK, 139-140) Kinder können noch nicht 
sprechen, aber sie versuchen, sich die Sprache anzueignen. Auch kennen sie noch nicht die Aktivität des Denkens, da es, nach Schleiermacher, kein Denken ohne Wörter gibt. Die Frage ist nun, wo sie anfangen sollen. Sie haben keinen einzigen Bezugspunkt. Wenn sie sich einige Wörter angeeignet haben, dann können sie durch Vergleich schnell andere Wörter lernen. Doch bleibt die Frage: Wie erwerben sie das erste Wort? Diese erste Fixierung ist für Schleiermacher ein divinatorisches Moment, und zwar des Ahnens oder Spekulierens, das am Anfang des Sprechens steht. Am Beispiel des Erlernens einer Sprache offenbart sich Schleiermacher die menschliche Seele als ein durch und durch spekulierendes und ahnendes Wesen. Wörter oder Denken, Sprache oder Begriff - man kann nicht sagen, was das erste ist; der erste Schritt ist ein Sprung.

Gadamer verweist auf Schleiermachers Interpretation der Situation des Kindes beim Erlernen der Sprache (GW 4, 364), verbindet es jedoch mit dem Gegensatz von allgemeiner Identität und subjektiver Individualität: "Solche relativen Nuancierungen bestätigen, dass absolut gesehen der Gegensatz von individuellen und Identischem jede Sprachsituation beherrscht." (GW 4, 364) Gadamer sieht die Spannung zwischen Identität und Individualität, und er sieht die Individualität der Sprache, die mit Hilfe der grammatischen Hermeneutik verstanden werden soll. Aber er versteht Schleiermachers Sprachauffassung "als auf die Identität der Vernunft hin gerichtet [...], wie diese in der Dialektik erworben werden soll". (GW 4, 369) Damit verkennt Gadamer, dass die Ungewissheit, die es zwischen Wort und Begriff, Sprache und Vernunft gibt, laut Schleiermacher nie in einem Verstehen aufgelöst werden kann, da jedes Verstehen an die Sprache gebunden bleibt.

Für Schleiermacher lebt der Mensch fortwährend in einer Situation der Unsicherheit, wenn auch auf einem kleinen und niedrigen Niveau. Auf dem Boden gemeinschaftlichen Wissens stehend, bleibt immer etwas Fremdes an der Sprache, die wir vom anderen hören und vernehmen. Wir haben es immer mit Unsicherheiten zu tun, auf die wir mit demselben divinatorischen 'Wissen' reagieren müssen. Immer muss der Geist sich selbst seinen Weg suchen, da jeder in seinem eigenen und individuellen Sein das Nicht-Sein des anderen ist; in dieser Hinsicht wird das Missverstehen nie verschwinden. Dies bedeutet für Schleiermacher, dass die Sprache ein vollständiges und unmittelbares Verstehen des Anderen verhindert.

Das Verstehen des Wortes ist möglich, wenn es als Teil eines Ganzen genommen wird. Jedes Kapitel eines Schriftwerkes muss im Kontext des Werkes als einer Ganzheit verstanden werden. Umgekehrt ist es erforderlich, das Ganze zu kennen, von dem aus man den einzelnen Abschnitt verstehen 
kann. Dennoch bleiben große Unsicherheiten. Man zieht vorläufige Schlüsse, zu denen man durch erste Orientierungsansätze gelangt, ohne die Unsicherheiten restlos zu beseitigen. Dieses Hin- und Zurückgehen vom Teil zum Ganzen kann in einem bestimmten Augenblick zum Stehen kommen, wobei am Ende vermutlich das volle Licht der Wahrheit auf die Dinge strahlt und alles vollends verstanden ist. Schleiermacher beschreibt diesen Augenblick des vollkommenen Lichtes als etwas Zufälliges, Unerwartetes, ähnlich dem Augenblick der ersten Fixierung von Wort und Denken.

Doch solange wir nicht das Ganze der Bedeutung eines Textes begriffen haben, brauchen wir eine ahnende Antizipation. (HK, 144) Hierfür sind ein Vorwort und eine Einleitung hilfreich, aber auch das Durchblättern eines Buches oder eine erste Lektüre des letzten Kapitels ist von Nutzen. Dennoch bleibt jede Antizipation unvollständig, solange bis wir vielleicht an dem Punkt des Verstehens angelangt sind, wo es keiner Hermeneutik mehr bedarf. Das Ganze der Bedeutung eines Textes, einer Rede zu begreifen, fällt nicht mehr in den Bereich der Hermeneutik. In der Hermeneutik ist jede Lösung lediglich eine Annäherung, da es unmöglich ist, das divinatorische Wissen durch ein demonstratives oder komparatives Wissen zu ersetzen. Dies gilt sowohl für die grammatische als auch für die psychologische Hermeneutik. Das Divinatorische bleibt notwendig, da die Kluft zwischen Wort und Begriff nie mit einem demonstrativen Wissen überbrückt werden kann.

\section{Hermeneutik und Dialektik}

Dass es in Schleiermachers Hermeneutik nicht allein um ein vernünftiges Verstehen geht, sieht Gadamer sehr wohl. Ebenso erkennt er, dass Schleiermacher sein Hauptaugenmerk auf das Verstehen von Sachverhalten richtet. Es geht nicht um die Intention des Autors, sondern um das Verstehen der "Sache". Die Hermeneutik ist der Sachforschung verpflichtet. Aus diesem Grund bezieht Schleiermacher, nach der Meinung Gadamers, die Hermeneutik auf die Dialektik. (WuM, 174) Gadamer ist sich der Bedeutung der Dialektik für Schleiermachers Hermeneutik bewusst, aber es scheint, als entschuldige er sich dafür, keine brauchbare Ausgabe dieser Dialektik zu haben. So schreibt er 1968: "Insbesondere seine [Schleiermachers - B. V.] Beiträge zur Hermeneutik sind stark beschnitten, und was hermeneutisch am meisten interessiert, seine Bemerkungen über Denken und Sprechen stehen überhaupt nicht in der 'Hermeneutik', sondern in seiner Dialektik-Vorlesung. Auf eine brauchbare kritische Ausgabe der Dialektik warten wir aber noch 
immer umsonst."6 Erst 1985 erkennt Gadamer, dass es eine verwandtschaftliche Nähe zwischen seiner Hermeneutik und der Schleiermachers gibt, und zwar in dem Punkt, wo bei Schleiermacher Dialektik und Hermeneutik ineinander übergehen: "Darin liegt am Ende die Rechtfertigung für die romantische Einsicht, der ich gefolgt bin, dass alles Verstehen schon Auslegen ist. Schleiermacher hat es einmal ausdrücklich gesagt: 'Das Auslegen unterscheidet sich von dem Verstehen durchaus nur wie das laute Reden von dem inneren Reden'."7 Ist die Auslegung als die Veräußerlichung des Verstehens anzusehen, so ist sie für das Gespräch ebenso von Bedeutung wie für die Hermeneutik. Das Gespräch ist in der Hermeneutik und der Dialektik von zentraler Bedeutung. Bei Schleiermacher ist die Hermeneutik auf die Dialektik als eine Theorie des Gesprächs bezogen. Im Gespräch lernt man seine Gedanken in Worte zu fassen und auszudrücken. Insofern ist Dialektik zu verstehen als die Theorie des zur Sprache-Bringens und in Worte-Fassens von Gedanken. Die Hermeneutik verfährt insofern anders, als sie die Wörter auf die Gedanken zurückführt ${ }^{8}$, wie in Schleiermachers Auslegung der Hermeneutik sichtbar geworden ist. Daher ist Hermeneutik nicht nur eine philologische, sondern auch eine philosophische Disziplin, mit deren Hilfe man Gedanken rekonstruiert. In dieser Hinsicht sind Dialektik und Hermeneutik beide Teil des Gesprächs und wechselseitig als philosophische Disziplinen aufeinander bezogen; die eine ist eine Lehre über die Versprachlichung der Gedanken, die andere eine Lehre über das Rekonstruieren der Gedanken aus Wörtern oder Texten.

Schleiermacher definiert Dialektik als die Lehre von den Prinzipien des Gesprächs. (D, 5) ${ }^{9}$ Motor des Gesprächs ist die Bewegung von den Unterschieden zur Einheit und Wahrheit in Bezug auf den Gegenstand. Konstituierende Bedingung für das Gespräch ist die Verschiedenheit der Sprache einerseits und die mögliche Einheit des Denkens andererseits. Auf dem Gebiet der Dialektik gibt es allerdings keine absolute Erkenntnis als Ideal der Einheit im Denken. In gewissem Sinn gibt es überhaupt keine Erkenntnis; jede endliche Erkenntnis ist immer der Anfang für eine neue dialektische

${ }^{6}$ H.-G. Gadamer, Klassische und philosophische Hermeneutik, GW 2, 92-117, hier 98.

${ }^{7}$ Ders., Zwischen Phänomenologie und Dialektik. Versuch einer Selbstkritik, GW 2, 3-23, hier 19.

${ }^{8}$ Diese Beziehung zwischen Hermeneutik und Dialektik wurde vor allem gesehen und entwickelt von Reinhold Rieger, Interpretation und Wissen. Zur philosophischen Begründung der Hermeneutik bei Friedrich Schleiermacher und ihrem geschichtlichen Hintergrund, Berlin/New York 1988.

${ }^{9}$ Friedrich Schleiermacher, Dialektik. Im Auftrag der Preußischen Akademie der Wissenschaften auf Grund bisher unveröffentlichten Materials herausgegeben von Rudolf Odebrecht, Darmstadt 1988, Unveränderter reprografischer Nachdruck der Leipziger Ausgabe von 1942 (abgekürzt als D). 
Auseinandersetzung, da gegen jede Behauptung immer wieder ein neuer Vorschlag vorgetragen werden kann. Der dialogische Charakter der Erkenntnis lässt keine definitive Antwort bzw. Lösung zu. Es gibt eine unauflösliche Relativität in dem Sinn, dass alles auf alles bezogen ist. Dies führt jedoch nicht zum Skeptizismus, da das Gespräch seinen Sinn aus dem Ideal der vollkommenen Erkenntnis ableitet.

Neben diesem Ideal ist das Gegenüber, die Stellung des Anderen und seine Ansicht(en) für die Bewegung des Denkens konstitutiv. Ohne diese Spannung könnte das Denken nicht in Bewegung versetzt werden. Der Konflikt ist immer auf die Wahrheit über etwas bezogen. Das erste Objekt des Denkens ist formal als Sein bestimmt; im Denken ist dieses Sein zunächst einmal leer. Seine erste Bestimmtheit erhält es erst durch das Gespräch über. (D, 23) Das Vorverständnis von der Identität des Seins, das das Subjekt besitzt, muss sich im Gespräch beweisen und bewahrheiten.

Vor dem Hintergrund dieser Überlegungen ist es befremdend, dass Gadamer in "Wahrheit und Methode" behauptet, dass es Schleiermacher nicht auf das Verhältnis zur Sache, d. h. des Seins ankommt. Was Schleiermachers Hermeneutik vorantreibt, seien ästhetische Motive: "Wo nun das Reden Kunst ist, da ist es auch das Verstehen. Alle Rede und aller Text sind also grundsätzlich auf die Kunst des Verstehens, die Hermeneutik, verwiesen, und so erklärt sich die Zusammengehörigkeit von Rhetorik (die ein Teilbereich der Ästhetik ist) und Hermeneutik." (WuM, 176 f.) In der Tat gibt es eine Verbindung von Rhetorik und Hermeneutik bei Schleiermacher, die jedoch nicht - wie Gadamer vermutet - ästhetisch motiviert ist. Die Rhetorik fällt in den Bereich der Dialektik, insofern diese als eine Lehre über die Versprachlichung der Gedanken zu verstehen ist.

Gerade weil das Denken nie eine absolute Identität der Gegensätzlichkeiten erreicht und sich immer zwischen Identität und Unterschied bewegt, ist dem Erkennen eine unhintergehbare Vorläufigkeit eigentümlich, die durch die individuelle und geschichtliche Weise ausgelöst wird, in der das Denken Gestalt annimmt, wird doch der Begriff in Form der Sprache, der Vorstellung und des Bildes dargestellt. Darum gehört zu jeder Universalität auch die Individualität des geschichtlichen Sprechens. Dieses Faktum, dass der Begriff immer in eine konkrete geschichtliche Aussage eingebettet bleibt, ist der Grund dafür, dass kein einziger Begriff eine absolute Erkenntnis verkörpert, sondern immer endlich und vorläufig ist.

Darum hat Erkenntnis einen hypothetischen Charakter; sie ist ein Vorschlag in Relation zum Gegenstand, auf den sie sich bezieht. Die Hypothese, die eine Annäherung an die angestrebte und gesuchte Identität ist, entspringt der 
Vermutung (Divination). (D, 75, 327) Das Vermuten wird durch die Phantasie vorangetrieben, die ihrerseits durch das Gefühl gelenkt wird, die der Ort der Einheit des Subjekts mit dem Ganzen der Wirklichkeit ist. Für Schleiermacher liegt der Zugang zur Einheit der Welt im Gefühl, nicht im Denken oder Wollen.

Da die Sprache das Medium des Denkens ist, ist die Einheit des Universums nicht denkbar. Die Sprache steht in einer Beziehung zum Universellen und Individuellen. Als System von Unterschieden und Unterscheidungen ist die Sprache immer auf eine individuelle Weise geordnet. In dieser Hinsicht sind alle Sprachen im Verhältnis zueinander irrational. Insofern eine Sprache individuell ist, stimmt sie mit einer anderen Sprache nicht überein. Übersetzen ist daher nur möglich durch den Gebrauch eines gemeinschaftlichen Denkens. Denken aber ist nur universell, sofern es in einer gemeinsamen Sprache besteht.

Demnach besitzt die Sprache auch einen universellen Aspekt, da wir uns ansonsten nicht verstehen würden können. In dieser Hinsicht ist jede Versprachlichung von Einsichten ein permanenter Versuch herauszufinden, ob alle Menschen ihre Gedanken auf die gleiche Weise konstruieren. (D, 373) Die Sprache ordnet alle individuellen Dinge durch Abstraktion in einem Schema, aus dem ein Begriff wird. Da der Begriff sich immer in einer Sprachhülle befindet, ist der Begriff die ideale Seite des Wortes. Einerseits macht die Sprache Unterscheidungen und individualisiert sie; andererseits universalisiert sie, sofern es eine gemeinsame Sprache gibt.

So gesehen ist die Sprache Ausdruck des Denkens, das in der Sprache an Klarheit und Schärfe gewinnt. (D, 127) Als solches steht es im Gegensatz zum Gefühl; durch die Sprache wird das ausgedrückte und in Worte gefasste Gefühl nicht vollständiger, sondern abgeschwächt. In gewisser Hinsicht scheinen Denken und Sprechen identisch zu sein. Sie unterscheiden sich jedoch dadurch, dass das Denken auf Universalität und Identität zielt, während das Sprechen sich in einer individuellen geschichtlichen Sprache vollzieht. Dieses Problem kann nicht durch eine universelle Sprache gelöst werden, da diese immer individuell und geschichtlich sein wird. (D, 16)

Der Mensch vermag es, sich dieser Relativität und Vorläufigkeit der Erkenntnis bewusst zu werden. (D, 327) Er kann dem Rechnung tragen und in Form von Regeln Vorsichtsmaßnahmen ergreifen. Gerade das Unvermögen, durch das Denken eine vollkommene Erkenntnis zu erlangen, führt zu der Notwendigkeit genauer Regeln, die es braucht, um jemanden wirklich verstehen zu können. Durch diese Regeln wird das Individuelle festgestellt und werden seine Grenzen markiert. Das Grundmotiv der Hermeneutik ist die 
allgemeine Unsicherheit in Bezug auf das Individuelle: "Wir müssen die individuelle Differenz selbst erkennen und bleiben also in unserer Aufgabe, nämlich im Wissenwollen [...] Und dies sind Gegenstände, von denen wir wissen, dass wir sie immer nur in der Approximation erreichen können." (D, 378) In diesem Gedankengang ist Gadamer Schleiermacher nicht gefolgt, wird doch seiner Meinung nach ein vollständiges Verständnis durch die Divination angestrebt: "Das zeigt sich daran, daß er so etwas wie ein vollständiges Verständnis annimmt, wenn die divinatorische Transposition einsetzt: 'bis dann am Ende erst wie auf einmal alles einzelne sein volles Licht erhält." (WuM, 179; HK 144) Meiner Meinung nach ist das Divinatorische in keiner Weise eine Garantie für ein vollständiges Verständnis. Die Leistung der Divination besteht darin, den Menschen bewusst werden zu lassen, dass er ein durch und durch ahnendes Wesen ist: "Dieses also ist das Ursprüngliche und die Seele bewährt sich auch hier als ganz und eigentlich ein ahndendes Wesen." (HK, 140) Zu recht folgert Gadamer: "Gewiss hat doch Schleiermacher die Individualität als ein nie ganz aufschließbares Geheimnis angesehen." (WuM, 179) Die Schranke zwischen Wort und Begriff soll durch das Gefühl durchbrochen werden. Aber dieses Gefühl ist keine Garantie für die Objektivität und Richtigkeit der Interpretation. Es gibt nur eine subjektiv gefühlte Ganzheit, die Unsicherheit der Interpretation bleibt. Dieses Problem hat Gadamer vor Augen, wenn er schreibt: "Schleiermachers Problem ist nicht das der dunklen Geschichte, sondern das des dunklen Du." (WuM, 179) Der Andere bleibt letzten Endes als Individuum unverständlich.

Dass ein vollständiges Verstehen bei Schleiermacher ein Ideal ist, ist von Gadamer erst später erkannt worden. Wäre ein solches Verstehen das Ideal der Hermeneutik, gebe es keine wirkliche Hermeneutik. Es handelt sich aber nur um ein Ideal, denn "das Nichtverstehen [ist] niemals gänzlich auf[zu]lösen". (HK 141; GW 4, 365) Gadamer neigt dazu, dieses Nichtverstehen psychologisch zu fassen: "Individualität ist ihrem Wesen nach in der Allgemeinheit des Gemeinschaftlichen nie ganz auflösbar. Daher ist auch im Verstehen der Menschen untereinander selbst für die empfänglichste Seele Fremdheit unaufhebbar, 'weil jede in ihrem einzelnen Sein Nichtsein der anderen ist"”. (GW 4, 365; HK, 141) Gadamer versteht dieses Problem nicht von der Divination her, wie Schleiermacher, sondern vom Gegensatz zwischen grammatischer und psychologischer Hermeneutik. Die Divination hat mit einer empfänglichen Seele nichts zu tun. Es handelt sich bei Schleiermacher nicht um die Originalität einer Seele, sondern um die nicht zu überbrückende Kluft zwischen Sprache und Denken. Trotzdem bleibt Gadamer bei seinem Ausgangspunkt: "Der Normbegriff, der hier das Verstehen leitet, ist 
das Originelle, und die Interpretationsweise ist die psychologische, die erklärt, wie aus dem Lebensganzen der Individualität diese originelle Rede sich herleitet." (GW 4, 365)

Auch ein Text bleibt das Resultat einer individuellen Sprache; die Universalität des Denkens erhält eine empirische individuelle Form im Text ${ }^{10}$ und ist letztendlich nicht in ihrer Ganzheit zu verstehen. Aber der Text ist auch durch die Interpretation zustande gekommen. Das Moment der Interpretation ist bereits bei der Konstitution des Textes vorhanden. Man kann schließlich nicht korrekt denken, ohne verständlich sein zu wollen. Denken wird gemeinschaftlich und vollständig, wenn es mitteilbar ist. $(D, 126)$ Nur in diesem Fall kann man von "Erkenntnis" sprechen. (D, 90) Daher muss der Schriftsteller sich die Frage stellen, wie er interpretiert werden will. Der andere, der Leser und Interpret, ist bereits bei der Konstitution des Textes präsent, weil der Schriftsteller vom Leser verstanden werden will. Schleiermacher betont, dass ein Text umso vollständiger ist, je dialogischer er aufgebaut ist. (D, 53 f.) Ein Text ist auch dann dialogisch, wenn es nur einen Autor gibt: dadurch nämlich, dass der Schriftsteller, indem er ständig danach fragt, ob er das, was er sagen will, auch wirklich geschrieben hat, sein erster Leser ist. Er wird den Text korrigieren und seine Sätze umformulieren, ausgehend von dem, was er über den Sachverhalt, um den es ihm geht, sagen will.

In diesem Sinne versteht Gadamer Schleiermachers Einsicht, dass es darauf ankommt, einen Schriftsteller besser zu verstehen als er sich selber verstanden hat. (WuM, 180 $)^{11}$ In Gadamers Verständnis bedeutet dies, dass Schleiermacher den Akt des Verstehens als den rekonstruktiven Vollzug einer Produktion ansieht, durch den bewusst gemacht werden soll, was dem Autor unbewusst geblieben ist. Es sei diese Intention, die Schleiermachers Hermeneutik in die Nähe zur Genieästhetik rücke. (WuM, 180) Diese These Gadamers lässt sich aus meiner Sicht nicht vereinbaren mit seiner Prämisse, dass der Künstler, der ein Gebilde schafft, nicht der berufene Interpret desselben ist, sondern - sofern er das von ihm Geschriebene selbst reflektiert - sein eigener Leser. Die Meinung, die er als Reflektierender hat, ist nicht maßgebend. Maßstab der Auslegung ist allein, was der Sinngehalt seiner Schöpfung ist. (WuM, 181) Nun ist aber die unbewusste Meinung des Autors das, was verstanden werden soll. Was Schleiermacher in seiner paradox

\footnotetext{
${ }^{10}$ Rieger, a. a. O. 286.

11 Wörtlich heißt es bei Schleiermacher: "Einen Autor besser zu verstehen als er selbst von sich Rechenschaft geben könne." HK, 138.
} 
anmutenden Formel sagen will, wird von Gadamer als Sachkritik verstanden: "Wer das, worüber der Autor spricht, besser zu durchdenken weiß, der wird das, was der Autor sagt, im Lichte einer ihm selbst noch verborgenen Wahrheit zu sehen vermögen." (WuM, 183)

Gadamer vermutet, dass Schleiermacher diesen Grundsatz philosophischer Kritik zu einem der philologischen Auslegungskunst umgedeutet hat - ohne zu sehen, dass für Schleiermacher die Hermeneutik letzten Endes eine philosophische Kritik ist. Aus diesem Grund macht es wenig Sinn, Schleiermacher vorzuwerfen, eine universelle Hermeneutik geschaffen zu haben, die "die vom Sachverständnis geführte Kritik aus dem Bereich der wissenschaftlichen Auslegung" herausdränge. (WuM, 184) Diese Kritik trifft nicht Schleiermacher. Es ist ein Missverständnis von Gadamer zu sagen: "Die Sprache ist ein Ausdrucksfeld, und ihr Vorrang im Felde der Hermeneutik bedeutet für Schleiermacher, daß er als Interpret die Texte unabhängig von ihrem Wahrheitsanspruch als reine Ausdrucksphänomene ansieht." (WuM, S 184) Wie gezeigt worden ist, ist der Bereich der Dialektik vom Ringen nach Wahrheit charakterisiert. Auch hier macht es sich negativ bemerkbar, dass Scheiermachers Dialektik kaum Einfluss auf Gadamers Urteil hat, obwohl er um die Bedeutung der Dialektik für die Hermeneutik Schleiermachers weiß. Gadamer sieht das Verdienst von Schleiermacher in einer Universalisierung der Hermeneutik, die letzten Endes aber nur methodisch motiviert sei, insofern er mit der Universalisierung eine gleichartige Methode für klassische und theologische Texte fand. (WuM, S. 185)

Für Schleiermacher sind Dialektik und Hermeneutik aufeinander bezogen wie Textkonstitution und -interpretation. Die Kunst des Sprechens und des Verstehens sind einander in dem Sinn gegenübergestellt, dass sie sich wechselseitig implizieren. Sie sind aufeinander bezogen wie Hören (Hermeneutik) und Sprechen (Dialektik). Diese Interaktion geschieht wie eine Bewegung vom Universalen zum Individuellen und vom Individuellen zum Universalen. Die erreichte Identität der Erkenntnis ist immer partiell, da sie durch die Textualität und den verbalen Charakter des Denkens an Unterschied und Individualität gebunden bleibt.

Die Bewegung vom Gedanken zum Wort ist auf die Dialektik bezogen, die Bewegung vom Wort zum Gedanken auf die Hermeneutik. Diese beiden ergänzen sich nicht nur, sie benötigen sich auch gegenseitig. Die Dialektik braucht die Hermeneutik, da die Identität des Erkannten nie ohne das Interpretieren der Sprache und der Wörter, die zu einer Identität gebracht werden müssen, gefunden werden kann. Andererseits braucht die Hermeneutik die Dialektik, da Sprache und Text nur verstanden werden können, wenn in ihnen 
eine Identität angenommen werden kann; sonst wäre die gesprochene Sprache unzugänglich.

Wie wir sehen, ist Dialektik hermeneutisch und Hermeneutik dialektisch. Mit anderen Worten: Die Bewegung vom Denken zum Sprechen wiederholt sich in der Bewegung von der Dialektik zur Hermeneutik. Vor diesem Hintergrund unterscheidet Schleiermacher zwei Arten von Denken: ein Denken, das von der Identität aller denkenden Subjekte ausgeht und ein Denken, das von den Unterschieden zwischen ihnen ausgeht. (D, 165) Durch die Dialektik löst sich jede Art der Erkenntnis in individuelle Unterschiede auf. Die Hermeneutik ist sich dessen bewusst und hebt die Dialektik in eine neue Universalität auf. Der hermeneutische und dialektische Prozess im Gespräch ist für die Bedeutung der Erkenntnis deshalb konstitutiv, weil er uns bewusst macht, dass eine absolute Identität unerreichbar, allenfalls annäherungsweise möglich ist, da immer ein Rest bleibt, der nicht begriffen werden kann.

Wir können sagen, dass ein Text verstanden ist, wenn seine unableitbare Identität (an)erkannt ist und wenn seine teilweise vorhandene Nichtverstehbarkeit akzeptiert wird. In diesem Sinne korrigiert die Hermeneutik die Dialektik, die auf die Einheit des Gesprächs gerichtet ist. Dialektik hat ihren Ort im Ideal universeller Identität, fällt jedoch ungewollt in den Bereich individueller Irrationalität. Die Hermeneutik setzt diese individuellen Unterschiede voraus. (D, 378) Gerade weil sie von diesen irreduziblen Unterschieden ausgeht, verweist die Hermeneutik auf den vorläufigen Charakter all jener Lösungen, die im Gespräch erzielt werden.

Die Hermeneutik bezieht die Dialektik auf die Geschichte und vervollständigt auf diese Weise die Erkenntnis. Sie lässt Vielheit und Unterschied zu. (D, 378) Die Gebundenheit der Erkenntnis an die Geschichte zeigt die Individualität der Erkenntnis. Dieser individuelle Charakter der Erkenntnis kann nie in Begriffen, sondern nur in Bildern und Anschauungen, wie z. B. Märchen, Erzählungen und Romanen, erfasst werden (D, 380), die nie zum Begriff werden können.

\section{Die Endlichkeit der Hermeneutik}

Laut Gadamer hat Schleiermacher seine Position nicht konsequent durchdacht: "Weder Schleiermacher noch Humboldt haben ihre Position wirklich zu Ende gedacht. Sie mögen die Individualität, die Schranke der Fremdheit, die unser Verstehen zu überwinden hat, noch so sehr betonen, am Ende findet doch lediglich in einem unendlichen Bewusstsein das Verstehen seine Vollendung und der Gedanke der Individualität seine Begründung. Es ist die 
pantheistische Eingeschlossenheit aller Individualität ins Absolute, die das Wunder des Verstehens ermöglicht." (WuM, 325) Worauf bezieht Gadamer seine Kritik? Meint er, dass Schleiermacher die Endlichkeit der menschlichen Erkenntnis nicht gesehen hat? Kritisiert er Schleiermacher, die Vorläufigkeit des hermeneutischen Unternehmens zuwenig beachtet zu haben?

Auch hier gilt, dass Gadamer Schleiermachers Dialektik zuwenig beachtet hat. Die Theorie der endlichen Erkenntnis hat Schleiermacher in seiner Dialektik entwickelt. ${ }^{12}$ Endliche Erkenntnis kommt durch das Kombinieren von Gegensätzen zustande. Die dabei anwesende Bezogenheit auf Identität findet im Gespräch statt, in dem die Gegensätze ausgedrückt und zu versöhnen gesucht werden. Das Ziel ist die höchste Erkenntnis, in der die Gegensätze versöhnt sind. Dieses Ziel kann man anstreben, es aber erst dann erreichen, wenn die Erkenntnis des höchsten Seins nicht durch Gegensätze und Unterschiede bestimmt ist. Daher kann es nicht durch die Sprache ausgedrückt werden und ist es dem von der Sprache abhängigen Subjekt nicht zugänglich. (D, 59) Allein die Erkenntnis, die im Kontrast und im Gegensatz zu etwas anderem steht, ist erreichbar. Unendlichkeit ist durch die Erkenntnis nicht erreichbar, bleibt aber als gefühltes Ideal im Hintergrund. In seinem Gefühl hat der Mensch ein "Bewusstsein" vom ganzen Universum: "Das Gefühl ist nur zurücktretend, nie ganz verschwindend". (D, 289) Wegen der Unerreichbarkeit des Unendlichen für das Denken mündet die Textkonstitution und das Gesprochene - als Ausdruck der Identität des Gesagten - in Opposition und im Unterschied zu Wörtern, während die Interpretation die Unterschiede und Gegensätzlichkeiten im Hinblick auf Bedeutungsfülle betrachtet.

Hermeneutik als Theorie des Verstehens gehört in den Bereich der Erkenntnis, nicht in den des Handelns oder Gefühls. Die Erkenntnis vollzieht sich in einem System von Zeichen, Wörtern und Sprache. Die Gemeinschaftlichkeit der Erkenntnis impliziert ihre Mitteilbarkeit, die der Sprache bedarf. Insofern ist Erkenntnis immer ausgedrückte Sprache oder Text, sogar dann, wenn die aktuelle intersubjektive Mitteilung nicht sichtbar ist, da jedes Subjekt als solches für sich selbst Gemeinschaftlichkeit durch das Mittel der Sprache zu erreichen sucht. Sprache hat daher einerseits den Charakter der Identität, sofern sie Ausdruck der Universalität der Erkenntnis ist; andererseits hat die Sprache den Charakter der Individualität, "weil jede Sprache in

\footnotetext{
12 Siehe hierzu auch: Ben Vedder, Was ist Hermeneutik? Ein Weg von der Texdeutung zur Interpretation der Wirklichkeit, Stuttgart 2000.
} 
sich selbst auf eigentümliche Weise modifiziert ist". (D, 376) Erkenntnis ist kein absolutes und zeitloses Phänomen, sondern existiert in einem geschichtlichen Kontext.

$\mathrm{Da}$ in jeder Erkenntnis etwas Individuelles liegt, das nicht mitteilbar ist, muss das Verstehen die Grenzen des Individuums durchbrechen; es muss etwas gefunden werden, durch das das Individuelle erschlossen wird. Dies geschieht nicht willkürlich, sondern vollzieht sich in der Analogie, die zwischen dem Fremden und Bekannten zu finden ist. Es ist das divinatorische Moment des Verstehens. Die Identität, die im anderen Individuum gefunden wird, ist allerdings nur im Gefühl anwesend, da nur im Gefühl die Bedeutungseinheit zu finden ist: "Vollständigkeit des Besonderen ist aber nie zu erlangen, also ist die Aufgabe eine unendliche. Wie kann die Vollständigkeit ersezt werden? [...] nur das Gefühl; und eben dies Gefühl muß auch die Vollständigkeit ersezen." (HK, 61) Das Gefühl ist ein Ersatz für das Verstehen. Das divinatorische Moment gründet sich im Gefühl; Gefühl und Divination sind komplementäre Begriffe: was im Divinatorischen offenbar wird, ist ein nicht mitteilbares Gefühl.

Information und Verstehen der Information setzen eine gemeinsame Identität voraus. Diese Voraussetzung kann nur durch ein Gefühl und nicht durch Erkenntnis garantiert werden. Denn absolute Erkenntnis, die ihren Platz nicht in einer geschichtlichen Wirklichkeit hat, ist einem endlichen, denkenden Subjekt ebenso wenig zugänglich wie eine absolute Identität.

Die endlose Interaktion von Denken und Sprechen und von Dialektik und Hermeneutik ist die Folge der Unmöglichkeit absoluter Erkenntnis und der Situierung der Erkenntnis im Bereich von Endlichkeit und Geschichte. Dass das Denken dies nicht bewältigen kann, bedeutet für das Denken einen unaufhebbaren Mangel: "Der Grund des Misslingens unserer Unternehmung liegt also darin, dass wir uns das Transzendente denken wollen." (D, 270) Das Denken vermittelt uns nie einen unmittelbaren und totalen Kontakt mit dem Universum; es blockiert letzten Endes das Verstehen des Ganzen. Und ein gefühltes Ganzes ist kein gedachtes Ganzes.

Das Verstehen richtet sich auf die Individualität des Textes, die nicht ganz individuell sein kann, da sie sonst nicht mitteilbar wäre. Das Wesen des Verstehens besteht darin, dass das Fremde, der Unterschied und das Individuelle in Bekanntes, in das Vertraute und Gemeinsame umgewandelt wird; dies geschieht durch die Anerkennung, nicht aber durch eine Beseitigung des Unterschieds, ist es doch die Aufgabe des Verstehens, die Fremdheit des Textes aufzuheben, ohne diese zu beseitigen. 
Die Individualität des zu verstehenden Objekts verlangt von dem, der es verstehen will, eine spezifische Betrachtungsweise, die in der Anwendung expliziter Regeln zum Ausdruck kommt. Die Bedeutung muss in einem Interpretationsprozess, der dem individuellen Charakter des Textes angepasst ist, zustande kommen. Um die Bedeutung eines Textes, der für uns aufgrund seiner Individualität nicht unmittelbar zugänglich ist, überhaupt erfassen zu können, haben die Regeln die Aufgabe, das Individuelle in Gemeinsames zu transformieren.

Das Individuelle ist jedoch durch Regeln nicht zu definieren, auch nicht durch die Regel des hermeneutischen Zirkels. Die unterschiedlichen Teile werden innerhalb des Ganzen verstanden, und das Ganze wird von seinen verschiedenen Teilen her verstanden, wobei das Ganze als Vorentwurf dient. Man hat sich jedoch das Ganze nicht zu Eigen gemacht, solange man die andere Individualität nicht ergründet hat. Das Ganze ist ein Rahmen, ohne den man die Teile nicht bestimmen kann. Dieses Ganze muss erfunden werden; wir brauchen einen Sprung zum vorausgesetzten Ganzen. Daher hat das Ganze die Struktur eines Vorentwurfs. Ohne Teile haben wir aber keinen Hinweis auf das Ganze.

Ganzes und Teil setzen einander voraus; diese Zirkularität im Verstehen basiert auf der wechselseitigen Priorität von Teil und Ganzem. (HK, 143) Würde das Individuelle aufgelöst werden, so verschwände die Polarität, und auch das Bedürfnis nach Hermeneutik wäre gestillt. Im Bereich des Denkens ist Verstehen eine endlose Bewegung, und es gibt niemals eine definitive Ausfüllung. Dadurch ist das Verstehen eine endlose Aufgabe. Verstehen ist eine Betrachtungsweise des Individuellen, nie eine Eliminierung des Individuellen zugunsten des Gemeinsamen; Beobachtung des Individuellen ist nie vollkommen und erschöpfend, sie kann immer korrigiert werden. Dies hat seinen Ursprung in der nicht ausschöpfbaren Individualität des Textes. Verstehen ist ein Teil der endlichen Erkenntnis, die keine absolute, sondern nur relative Sicherheit besitzt. Daraus folgt, dass die Bedeutung eines Textes nie definitiv feststeht und dass die Geschichte seiner Auslegung nie an ein Ende gelangt.

Dieser Prozess spielt sich innerhalb der Sprache ab: "Alles vorauszusehende in der Hermeneutik ist nur Sprache und alles zu findende [...] muß aus der Sprache gefunden werden." (HK, 38) Es mag symptomatisch scheinen, dass Gadamer nur die erste Hälfte dieses Satzes als Motto am Schluss des dritten Abschnitts von Wahrheit und Methode angibt. (WuM, 361) Schleiermachers These besagt, dass die Hermeneutik immer an der Sprache gebunden bleibt. Der objektive Aspekt der Sprache ist die Aufgabe der grammatischen 
Hermeneutik, der subjektive Aspekt ist die Aufgabe der psychologischen Hermeneutik. So sind grammatische und psychologische Hermeneutik aufeinander bezogen. Die Sprache ist einerseits durch ein Sprachsystem (objektiv) bestimmt, und andererseits durch den gedanklichen Inhalt des Autors (subjektiv). Diese beiden Aspekte der Sprache führen für die Hermeneutik zu zwei spezifischen Betrachtungsweisen.

Die grammatische Interpretation versucht, das Individuelle durch die verschiedenen Arten von Verbindungen in der Sprache zu verstehen; aus dem Vergleich der einzelnen Teile leitet man die Einheit der Bedeutung ab. Doch bleibt ein Rest, der nur durch das Gefühl gelöst werden kann. Dies bedeutet, dass auch in der grammatischen Hermeneutik für das divinatorische Moment ein signifikanter Platz ist. (HK, 155) Dieses Moment kann aber nur durch das Gefühl vervollständigt und abgerundet werden, das Gefühl aber steht außerhalb sowohl der Erkenntnis als auch des hermeneutischen Prozesses.

Die Textinterpretation ist sowohl von der grammatischen als auch der psychologischen Betrachtungsweise abhängig. Beide Betrachtungsweisen verwenden die komparative und die divinatorische Methode. Die komparative Methode vergleicht das Individuelle, sofern es etwas mit etwas anderem gemeinsam hat. Und die divinatorische Methode vergleicht das Individuelle, insofern es Unterschiede zum anderen gibt. Die zerbrechliche Sicherheit der Erkenntnis ist durch die Sicherheit des Gefühls ersetzt, gerade weil das Gefühl die Fähigkeit hat, ein individuelles Ganzes für sich zu komplettieren. Divination ist möglich und notwendig, weil jeder Mensch, ungeachtet der Tatsache, dass er selbst ein eigenes Individuum ist, für alle anderen Individuen im Gefühl empfänglich ist. Im Gefühl ist die Beziehung zum anderen wie auch zum Ganzen wiederhergestellt - im Unterschied zum Bereich des Denkens, wo der Unterschied und die Unüberbrückbarkeit zum Anderen und zum Ganzen dominiert.

Vergleich und Divination sind untrennbar miteinander verbunden, da die Divination ihre intellektuelle Sicherheit durch den Vergleich erhält, ohne den sie nur Phantasie wäre. Allein der Vergleich führt nicht zum Verstehen. Das Vergleichen hat keine Einheit ohne Divination, die den Zugang zur individuellen Ganzheit des Textes gewährt. Beide brauchen einander für das Verstehen eines Textes. Diese Zusammenarbeit ist nur möglich, wenn das Verstehen seine Endlichkeit akzeptiert und anerkennt, dass die Erkenntnis des Absoluten unmöglich ist und nur durch das Gefühl ersetzt werden kann. Eine 'Erkenntnis' des Absoluten ist nur jenseits der Endlichkeit möglich, nämlich im Gefühl des Ganzen.

Es war Gadamers Ansicht, dass Schleiermacher eine christliche Inspiration vermeiden wollte: "Durch die individualisierende psychologische Interpretation 
sucht er die alle philologischen und historischen Maßstäbe brechende Inspirationshermeneutik, die sich auf den Heiligen Geist beruft, auszuschalten." (GW 4, 373). Aber dieses Argument gilt nur, wenn man divinatorische und psychologische Methode in der Weise identifiziert, wie es Gadamer getan hat.

Ben Vedder (1948) studierte Theologie und Philosophie in Utrecht und Leuven. Er ist Professor für Metaphysik und Erkenntnislehre an der philosophischen Fakultät der Universität Nijmegen. Er publiziert besonders über Hermeneutik, Metaphysik und Religionsphilosophie. Auf Deutsch erschien von ihm: Was ist Hermeneutik? Ein Weg von der Textdeutung zur Interpretation der Wirklichkeit, Kohlhammer, Stuttgart, 2000.

Anschrift: Vierde Herven 2, $5232 \mathrm{JH}$ 's-Hertogenbosch. 